\title{
On the theory of tumor self-seeding: implications for metastasis progression in humans
}

\author{
Julio A Aguirre-Ghiso*
}

\begin{abstract}
Metastasis remains the leading cause of death among cancer patients because few effective treatment options are available. A recent paper proposes a new twist on metastasis. The paper shows that circulating tumor cells can return to the primary tumor, a process termed tumor self-seeding or cross-seeding, and that this helps breeding tumor cells that give rise to aggressive metastatic variants. A viewpoint presented here addresses the implications of these studies for human cancer metastasis.
\end{abstract}

\section{Background}

Cancer cells disseminate and lodge in target organs, producing a distinct and mostly predictable pattern of metastasis [1,2]. Metastatic cells may evolve in the primary tumor but also, following dissemination, at distant sites [1]. Numerous genes favoring or suppressing the multiple metastatic steps have been identified [1,2]. Moreover, primary tumor gene expression profiles revealed signatures that can predict poor treatment outcomes, most probably linked to metastasis development [3].

In spite of the progress, however, due to important confounding factors, the dynamics driving the emergence of metastatic variants in patients is poorly understood. First, patients presenting with non-invasive lesions (that is, ductal carcinoma in situ) already have disseminated tumor cells $[1,4]$. Second, the metastatic phenotype can be acquired in parallel in the primary lesion and in the organ-lodged disseminated tumor cells, and the latter may evolve after primary tumor surgery and without development of local recurrences [1]. Third, metastasis development can be remarkably delayed - suggesting that, before resuming growth and evolution, the lodged

*Correspondence: julio.aguirre-ghiso@mssm.edu

Department of Medicine and Department of Otolaryngology, Division of Hematology and Oncology, Tisch Cancer Institute at Mount Sinai, Mount Sinai School of Medicine, One Gustave L. Levy Place, Box 1079, New York, NY 10029, USA disseminated tumor cells (minimal residual disease) can remain dormant [5]. In a recent study, Kim and colleagues propose that the ability of circulating tumor cells (CTCs) to return to and grow in the primary tumor might aid in selecting the seeds of metastasis [6].

\section{Article}

Kim and colleagues at the Memorial Sloan Kettering Cancer Center asked whether CTCs could reseed a primary tumor [6]. Furthermore, they tested whether this reseeding selects for variants endowed with specific genes driving metastasis.

The authors tagged breast and melanoma cell lines with fluorescent proteins and/or luciferase. The MDA-MB-231 human breast carcinoma cell line (MDA231) and its variant, selected via intravenous inoculations for enhanced lung colony formation (MDA231-LM2) [7], were primarily used in reseeding or cross-seeding experiments. Tagged and untagged tumor cells were injected separately into orthotopic contralateral sites. MDA231-LM2 cells were highly efficient in disseminating and self-seeding a contralateral MDA231-LM2 or MDA231 mass or in cross-seeding breast MCF7 or melanoma A375 tumors. Similarly, MDA231-LM2 lung colonies produced by intravenous injection also seeded orthotopically growing MDA231 tumors. Thus, regardless of their growth location, the MDA231-LM2 cells can seed an established tumor mass.

Curiously, MDA231-LM2 cells were not reported to spontaneously seed lungs from the primary tumor. This suggests that spontaneous seeding of a tumor mass is less restrictive than seeding of target organs. Alternatively, enhanced lung colonization selected through forced intravenous inoculation [7] might exacerbate cell traits that do not entirely recapitulate organ-specific metastasis.

In an important experiment, MDA231 cells that spontaneously seeded the same contralateral tumor were enriched (MDA231-S1a). Upon reinjection through different routes, MDA231-S1a cells spontaneously seeded a contralateral tumor, but apparently not lungs or other organs. Only after direct injection into the bloodstream did they seed bones, brain, or lungs. IL- 6 and IL- 8 produced by contralateral human tumors served as CTC 
attractants, and this might explain the preferred spontaneous tumor versus lung targeting. The role of human tumor versus mouse lung-derived cytokines directing this tropism, however, cannot be ruled out. MMP1, FASCIN1 and CXCL1 genes required here for tumor seeding are also part of a human breast cancer signature associated with metastasis development [7]. Further, stromal cells aided self-seeders and the latter cells also accelerated primary tumor growth.

The identified genes or the stromal cell involvement are not entirely new to metastatic progression [2,8-11]. All organ-specific metastasis genes being represented in the MDA231-S1a derivate, however, was a surprising observation. Possibly only few of these genes are required for tumor mass colonization, and the additional organspecific genes are enriched because they facilitate but are not required for self-seeding. Alternatively, the contralateral tumor mimics a multiorgan microenvironment favoring selection of all variants simultaneously.

Overall, these studies show that important information on the mechanisms contributing to target organ colonization can be drawn from self-seeding or crossseeding experimental models.

\section{Viewpoint}

Is there any parallel between these experiments and metastasis in patients, and what clinical scenarios could be envisioned for such a process? These experiments possibly model a rare phenomenon in patients where one malignant tumor (for example, prostate or breast carcinoma) metastasizes to a different second primary tumor, usually a benign lesion [12-14]. These are exceedingly rare cases even in the 2 to $3 \%$ of patients that develop two different cancers simultaneously. Kim and colleagues' study shows that, as in these rare cases, malignant cells will metastasize to a second tumor if available. In these patients, however, cross-seeding was simultaneous with multiple organ metastasis [14].

Self-seeding and the presence of a tumor mass may not be a sine qua non requisite because metastasis develops in patients after undergoing surgery (the majority) for small or larger invasive lesions, such as in breast cancer. If tumor masses are required [6], self-seeding would not select for metastatic variants during periods of minimal residual disease or favor local recurrences, unless local microscopic residual disease or a remaining reactive stroma is sufficient to fuel this process. Self-seeding, however, may occur in patients with inoperable primaries and metastases or in those that, after primary tumor surgery, still carry multiple metastases and may have a constant CTC population. Finally, the timing of metastasis must be considered. Why in some patients does it take 1 or 2 years but in other patients 10 years or more for the metastatic variants carrying the poor prognosis genes $[3,7]$ to grow into overt metastasis? Does this mean that the time to overt metastasis is regulated by other mechanisms and that self-seeding or cross-seeding is only relevant between established metastasis [15]? These and many other questions generated by this thoughtprovoking study will surely fertilize the field of metastasis to provide a clearer view on how to target this lethal step of cancer progression.

Abbreviations

CTC, circulating tumor cell; IL, interleukin.

Competing interests

The author declares that he has no competing interests.

\section{Acknowledgements}

The author would like to thank colleagues and members of the laboratory for helpful discussions. JAA-G is supported by grants from the Samuel Waxman Cancer Research Foundation Tumor Dormancy Program, the National Cancer Institute (CA109182), the NIEHS (ES017146) and NYSTEM-ESSCB.

Published: 28 April 2010

\section{References}

1. Klein CA: Parallel progression of primary tumours and metastases. Nat Rev Cancer 2009, 9:302-312.

2. Nguyen DX, Bos PD, Massague J: Metastasis: from dissemination to organspecific colonization. Nat Rev Cancer 2009, 9:274-284.

3. van de Vijver MJ, He YD, van't Veer LJ, Dai H, Hart AA, Voskuil DW, Schreiber GJ, Peterse JL, Roberts C, Marton MJ, Parrish M, Atsma D, Witteveen A, Glas A, Delahaye $L$, van der Velde T, Bartelink H, Rodenhuis S, Rutgers ET, Friend SH, Bernards R: A gene-expression signature as a predictor of survival in breast cancer. N Engl J Med 2002, 347:1999-2009.

4. Husemann Y, Geigl JB, Schubert F, Musiani P, Meyer M, Burghart E, Forni G, Eils $R$, Fehm T, Riethmuller G, Klein CA: Systemic spread is an early step in breast cancer. Cancer Cell 2008, 13:58-68.

5. Aguirre-Ghiso JA: Models, mechanisms and clinical evidence for cancer dormancy. Nat Rev Cancer 2007, 7:834-846.

6. Kim MY, Oskarsson T, Acharyya S, Nguyen DX, Zhang XH, Norton L, Massague J: Tumor self-seeding by circulating cancer cells. Cell 2009, 139:1315-1326.

7. Minn AJ, Gupta GP, Siegel PM, Bos PD, Shu W, Giri DD, Viale A, Olshen AB, Gerald WL, Massague J: Genes that mediate breast cancer metastasis to lung. Nature 2005, 436:518-524.

8. Polette M, Clavel C, Birembaut P, De Clerck YA: Localization by in situ hybridization of mRNAs encoding stromelysin 3 and tissue inhibitors of metallo-proteinases TIMP-1 and TIMP-2 in human head and neck carcinomas. Pathol Res Pract 1993, 189:1052-1057.

9. Wyckoff J, Wang W, Lin EY, Wang Y, Pixley F, Stanley ER, Graf T, Pollard JW, Segall J, Condeelis J: A paracrine loop between tumor cells and macrophages is required for tumor cell migration in mammary tumors. Cancer Res 2004, 64:7022-7029.

10. Tlsty TD, Coussens LM: Tumor stroma and regulation of cancer development. Annu Rev Pathol 2006, 1:119-150.

11. Dhawan $P$, Richmond $A$ : Role of CXCL1 in tumorigenesis of melanoma. $J$ Leukoc Biol 2002, 72:9-18.

12. Doron Y, Gruszkiewicz J: Metastasis of invasive carcinoma of the breast to an extradural meningioma of the cranial vault. Cancer 1987, 60:1081-1084.

13. Bernstein RA, Grumet KA, Wetzel N: Metastasis of prostatic carcinoma to intracranial meningioma. Case report. J Neurosurg 1983, 58:774-777.

14. Sella A, Ro JY: Renal cell cancer: best recipient of tumor-to-tumor metastasis. Urology 1987, 30:35-38.

15. Engel J, Eckel R, Kerr J, Schmidt M, Furstenberger G, Richter R, Sauer H, Senn $\mathrm{HJ}$, Holzel D: The process of metastasisation for breast cancer. Eur J Cancer 2003, 39:1794-1806.

doi:10.1186/bcr2561

Cite this article as: Aguirre-Ghiso JA: On the theory of tumor self-seeding: implications for metastasis progression in humans. Breast Cancer Research 2010, 12:304. 